\title{
Hysteresis in human binocular fusion: temporalward and nasalward ranges
}

\author{
Daniel B. Diner* and Derek H. Fender \\ California Institute of Technology, Pasadena, California 91125
}

Received December 17, 1984; accepted April 14, 1987

\begin{abstract}
Fender and Julesz [J. Opt. Soc. Am. 57, 819 (1967)] moved pairs of retinally stabilized images across the temporalward visual fields and found significant differences between the disparities that elicited fusion and the disparities at which fusion was lost. They recognized this phenomenon as an example of hysteresis. In the work reported in this paper, binocular retinally stabilized images of vertical dark bars on white backgrounds were moved into horizontal disparity in both the nasalward and the temporalward directions. The limits of Panum's fusional area and the hysteresis demonstrated by these limits were measured for two observers. The following results were obtained: (1) the nasalward limits of Panum's fusional area and the hysteresis demonstrated by the nasalward limits do not differ significantly from the temporalward limits and the hysteresis demonstrated by the temporalward limits; (2) the limits of Panum's fusional area and the hysteresis demonstrated by these limits are not significantly different if one stimulus moves across each retina or if one stimulus is held still on one retina and the other stimulus is moved across the other retina; (3) the use of nonstabilized cross hairs for fixation decreases the hysteresis; and (4) the full hysteresis effect can be elicited with a rate of change of disparity of $2 \mathrm{arcmin} / \mathrm{sec}$.
\end{abstract}

\section{INTRODUCTION}

Wheatstone, ${ }^{1}$ Dove, ${ }^{2}$ and Panum $^{3}$ noted that exact alignment of the visual axes is not necessary for single binocular perception. The region over which fusion is possible is known as Panum's fusional area. Recent work has shown that the fusional area is not fixed but can change in certain circumstances. Fender and Julesz, ${ }^{4}$ using binocular retinally stabilized images, found that when the images of two bars were moved into correspondence and then slowly moved apart again, the images fused at one disparity and then lost fusion at a larger disparity. Hence they showed that Panum's fusional area can be changed as a function of its recent stimulation.

One of the stimuli used by Fender and Julesz 4 consisted of two dark bars, 13 arcmin $\times 60$ arcmin, on light backgrounds. These bars fused at $42 \pm 10$ arcmin and lost fusion at $65 \pm 14$ arcmin, as shown in Fig. 1.

A stabilized image cannot be moved on the retina by eye movements. Thus if a stabilized image is projected eccentrically from the central fovea, it will trigger a series of saccades, which drives the direction of gaze outside the range of the stabilization apparatus. Hence Fender and Julesz 4 moved the targets symmetrically into the temporalward visual fields only, since the human oculomotor system cannot diverge the visual axes more than a small angle. Hyson et $a l .5$ measured eye movements while studying extension of the fusional area in normal vision. Using a $10^{\circ}$-wide random-dot stereogram, they were able to measure extensions of up to $4.1^{\circ}$ of the temporalward fusional area. Neither of these research groups moved the targets into the nasalward visual fields, nor did they move the targets asymmetrically.

Erkelens and Collewijn ${ }^{6}$ also measured eye movements while studying the extension of the fusional area in normal vision. Using a $30^{\circ}$-wide random-dot stereogram, they were able to measure extensions of between $1^{\circ}$ and $2^{\circ}$ for differ- ent subjects. Although they moved the targets into the nasalward visual fields, they found substantial difficulties and did not report the resulting data. They did not move the targets asymmetrically.

Piantanida 7 employed a form of retinal image stabilization that is probably less accurate than that employed by Fender and Julesz. ${ }^{4}$ Using a $3^{\circ}$-wide random-dot stereogram or a 13-arcmin-wide and 61-arcmin-tall line stereogram, he measured both the temporalward and nasalward fusional ranges. He reported sums of these ranges between $1^{\circ}$ and $2.5^{\circ}$. He defined a preferred setting as "the horizontal offset ... producing the most comfortable fusion of the stabilized random dot stereogram." 7 Piantanida found that this point fell well to the nasalward side of the midpoint of the fusional and refusional ranges. He found, in contrast to the results obtained by all other researchers, that the refusional range was greater than the initial fusional range.

It has been shown that other parameters of the stimuli can influence the extent of the fusional area. For example, Kertes $z^{8}$ showed that increased stimulus size resulted in significant increases in horizontal and vertical fusional amplitudes. In addition, both Kulikowski ${ }^{9}$ and Schor et al. ${ }^{10}$ showed that the presence of high spatial frequencies in the stimulus reduced the size of Panum's fusional area. Furthermore, Schor and Tyler ${ }^{11}$ manipulated the spatiotemporal modulation of binocular disparity and concluded that the fusional area is a "combination of a constant minimum area with an extended area that responds to low frequency time-varying disparities." Our examination of their results leads us to believe that the maximum extension occurs at a combination of low temporal frequencies and low spatial frequencies.

In the studies of Schor et al. ${ }^{10}$ and Schor and Tyler, ${ }^{11}$ the experimenters employed visual stimuli selected to ensure that they were measuring the sum of nasalward and temporalward fusional disparities. However, they did not measure 


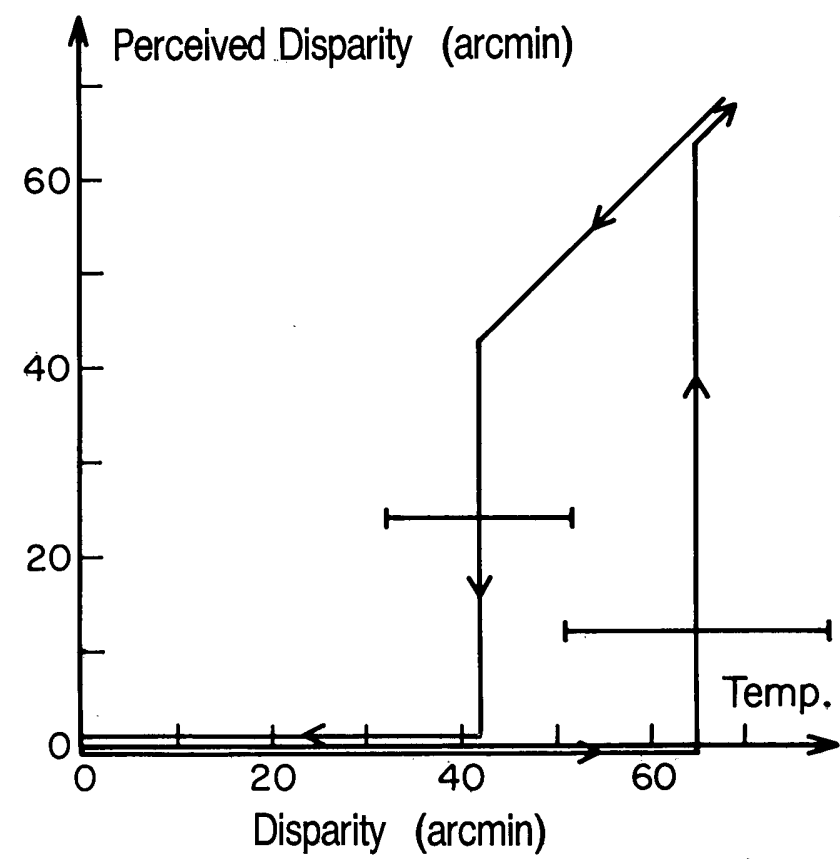

Fig. 1. Fusion and hysteresis for stabilized images of vertical bars as measured by Fender and Julesz. ${ }^{4}$ Temp., temporalward. Horizontal bars represent 1 standard deviation. Fixation reticules were not used. In this and the following figures, we assume that the perceived disparity is zero during fusion and that it is veridical in the absence of fusion.

eye movements, and thus they were unable to determine the relative magnitude of the nasalward versus the temporalward limits of fusion.

In the work reported in this paper we have measured both the temporalward and the nasalward limits of fusion by using stabilized retinal images and nonstabilized fixation cross hairs carefully placed in the straight-ahead direction for each eye.

\section{EXPERIMENTAL METHOD}

\section{Equipment}

In the experiments reported in this paper, the original equipment of Fender and Julesz ${ }^{4}$ was used, with the following differences:

(1) The targets were controlled by stepping motors, which moved each target 0.25 arcmin per step.

(2) The visual field was restricted to $3^{\circ}$ in diameter by a nonstabilized circular mask.

(3) A nonstabilized fixation reticule consisting of a pair of cross hairs with a 50-arcmin-radius ring was introduced into each visual field.

(4) The stimuli were identical to the bars used by Fender and Julesz, ${ }^{4}$ but the tops of the bars were aligned with the horizontal member of the fixation reticule, thus placing the bars in the lower visual field.

(5) Hydrostatic suction was used to minimize slippage of the contact lenses on the eyeballs of the observer. ${ }^{12}$

\section{Accuracy of Stabilization}

It is important to know the accuracy of the image stabilization; otherwise the movement of an imperfectly stabilized image could be confused with a shift of Panum's fusional area. Slippage of the contact lens is such a source of error. This was controlled as follows. If the stabilized image of a dark bar on a light background slips, the observer perceives a three-part bar caused by afterimage effects. The part of the bar image that moves into a region of retina light adapted by the background yields the percept of a dark bar. The light region of background that moves into the region of retina dark adapted by the bar yields the percept of a bright bar. The rest of the bar, projected upon the retina adapted for it, appears to be unchanged. This three-part bar percept is vivid, and an image slippage of 1 arcmin can easily be detected. The hydrostatic suction was set to a value equal to twice the pressure necessary to reduce the slippage to zero. If this pressure exceeded a safe value, a new contact lens was molded for a better fit.

\section{PROCEDURE}

\section{Initial Adjustments}

Each channel of the binocular retinal-image-stabilization apparatus was adjusted so that the center of the field of view of each channel was in the straight-ahead position and the optical axes of the two channels were parallel. A nonstabilized reticule was introduced into each channel and was adjusted so that it was located at the center of the field of view.

A stabilized image of a bar and a nonstabilized image of the reticule were presented to each eye in turn. The observer adjusted the image of each bar, while maintaining fixation on the reticule, until each bar was aligned with the corresponding reticule. The stimuli were then presented binocularly, and the positions of the stabilized bars were finely adjusted until the fused image of each bar hovered below and in the same depth plane as the center of the reticule.

\section{Experimental Regime}

The experiment was controlled by a computer, which moved the stabilized images in some predetermined manner. The observer was instructed to press a button whenever fusion was lost and to release the button when the bars appeared to be fused. Any button press or release caused the locations of the targets, measured by potentiometers accurate to 1 arcmin, to be recorded by the computer.

\section{Training}

The observers were first trained to overcome the foveation reflex. They were instructed to fixate the reticule while manually adjusting a stabilized bar to eccentric positions. Any eye motion caused the percept of a corresponding movement of the stabilized bar. Eye movements of the observer were monitored by the method described by St-Cyr and Fender. ${ }^{13}$ Only one monocular image was presented initially. Once the observer could maintain fixation during this task for either eye, the training continued with two stabilized bars viewed binocularly. When the observer could maintain fixation during this second task, the stabilized images were put under computer control and were moved at a 2-arcmin/sec disparity change until they reached 70 arcmin of crossed disparity. The motion was then reversed, the images were moved to 70 arcmin of uncrossed disparity, and so on. 


\section{THE EXPERIMENTS}

\section{Experiment 1}

The conditions were those of the last phase of training. The stabilized images were moved into the temporalward visual fields at $2 \mathrm{arcmin} / \mathrm{sec}$. When the observer pressed the response button signaling the loss of fusion, the positions of the bars were recorded. The binocular disparity between the bars thus measured is called the disparity at temporalward break in the rest of this paper. The motion of the bars continued to a disparity of 70 arcmin to ensure the decay of any neural adaptation corresponding to the extended fusional range. 4,5 The motion of the bars was reversed, and the observer released the button on perceiving the fusion of the bars. This value of binocular disparity is called the disparity at temporalward fusion. The motion of the bars was then continued into crossed disparity, giving a value for the disparity at nasalward break. The motion of the bars was continued to a nasalward disparity of $70 \mathrm{arcmin}$ and then reversed, giving a value for the disparity at nasalward fusion. The motion of the bars was continued until the percept of temporalward break, and the cycle was continued ad libitum.

\section{Experiment 2}

The reticules were removed, and the observers were instructed to fixate the center of the visual field as defined by the circular mask. All other conditions of the experiment were unchanged.

\section{Experiment 3}

The reticules were replaced, and the stabilized image of one bar was held still on one retina while the image of the other bar was swept across the other retina at $2 \mathrm{arcmin} / \mathrm{sec}$.

\section{RESULTS}

The results of experiments 1-3 are given in Table 1 and illustrated in Figs. 2 and 3.

There is no absolute zero of binocular retinal disparity; this position must be determined statistically, as was recognized by Ogle. ${ }^{14}$ For each experiment the nasalward fusion mean and the temporalward fusion mean were therefore averaged, and the resulting average was taken as the zero-disparity value. The mean values for disparities at nasalward break, nasalward fusion, temporalward break, and temporalward fusion were then adjusted around this zero value. The centering of nasalward fusion and temporalward fusion forces these means to be equal in each experimental condition (see Table 1). However, in the raw data, the mean value
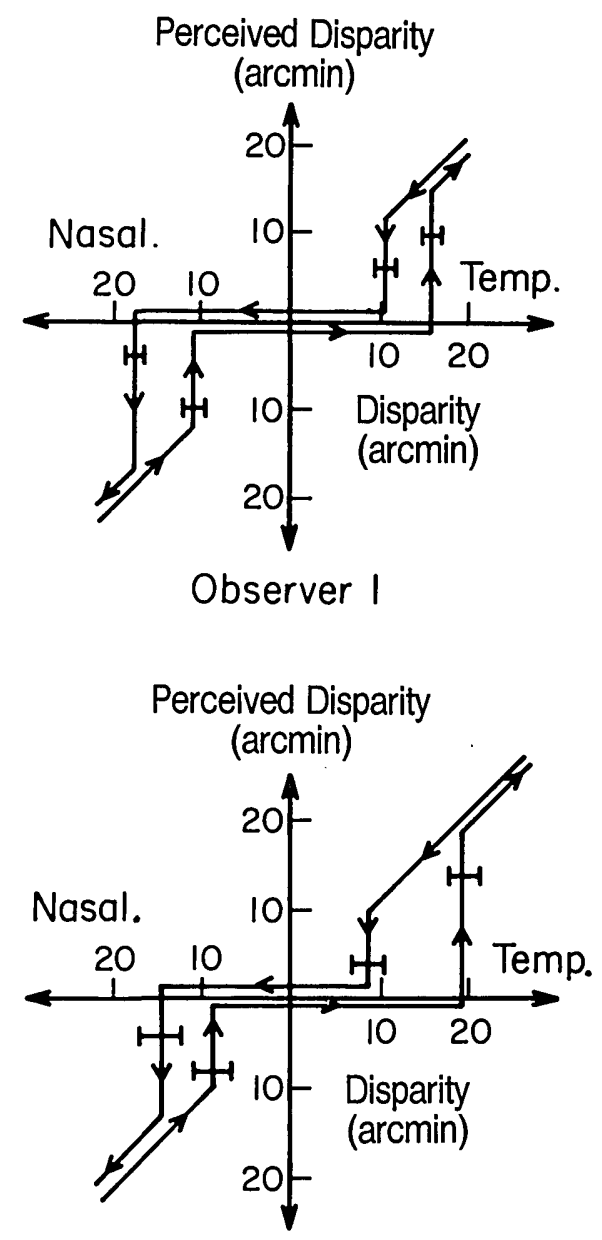

Observer 2

Fig. 2. Fusion and hysteresis for stabilized images of vertical bars, temporalward and nasalward ranges. Temp., temporalward; Nasal., nasalward. Horizontal bars represent 1 standard error of mean. Fixation reticules were used.

Table 1. Binocular Disparities for Fusion and Hysteresis in Stabilized Vision

\begin{tabular}{|c|c|c|c|c|c|c|}
\hline \multirow[b]{2}{*}{ Experiment $^{a}$} & \multirow[b]{2}{*}{ Observer } & \multicolumn{4}{|c|}{ Disparity $(\operatorname{arcmin})^{b}$} & \multirow{2}{*}{$\begin{array}{c}\text { Number of } \\
\text { Trials }\end{array}$} \\
\hline & & Nasal Break & Nasal Fusion & Temporal Fusion & Temporal Break & \\
\hline 1 & 1 & $-17.6 \pm 0.9$ & $-10.6 \pm 0.8$ & $10.6 \pm 0.9$ & $16.0 \pm 0.7$ & 23 \\
\hline 2 & 1 & $-21.8 \pm 0.9$ & $-9.6 \pm 0.9$ & $9.6 \pm 1.1$ & $21.1 \pm 0.9$ & 20 \\
\hline $3 a$ & 1 & $-15.4 \pm 2.1$ & $-10.6 \pm 1.2$ & $10.6 \pm 0.8$ & $14.8 \pm 1.0$ & 20 \\
\hline $3 b$ & 1 & $-16.7 \pm 1.4$ & $-9.4 \pm 0.8$ & $9.4 \pm 1.0$ & $14.0 \pm 0.9$ & 19 \\
\hline 1 & 2 & $-14.2 \pm 1.7$ & $-8.8 \pm 1.5$ & $8.8 \pm 1.2$ & $19.5 \pm 1.4$ & 28 \\
\hline 2 & 2 & $-26.6 \pm 1.4$ & $-14.8 \pm 1.4$ & $14.8 \pm 1.4$ & $23.6 \pm 1.7$ & 13 \\
\hline $3 a$ & 2 & $-19.3 \pm 0.8$ & $-8.6 \pm 1.0$ & $8.6 \pm 0.5$ & $15.9 \pm 1.0$ & 18 \\
\hline $3 b$ & 2 & $-17.3 \pm 0.8$ & $-12.1 \pm 0.9$ & $12.1 \pm 0.8$ & $19.1 \pm 0.7$ & 23 \\
\hline
\end{tabular}

\footnotetext{
a Conditions: experiment 1, both bars in motion, reticules used for fixation; experiment 2, both bars in motion, no fixation mark; experiment 3a, right bar in motion, left bar stationary, reticules used for fixation; experiment $3 \mathrm{~b}$, left bar in motion, right bar stationary, reticules used for fixation.
}

${ }^{b}$ Each entry represents the mean plus or minus the standard error of the mean. 

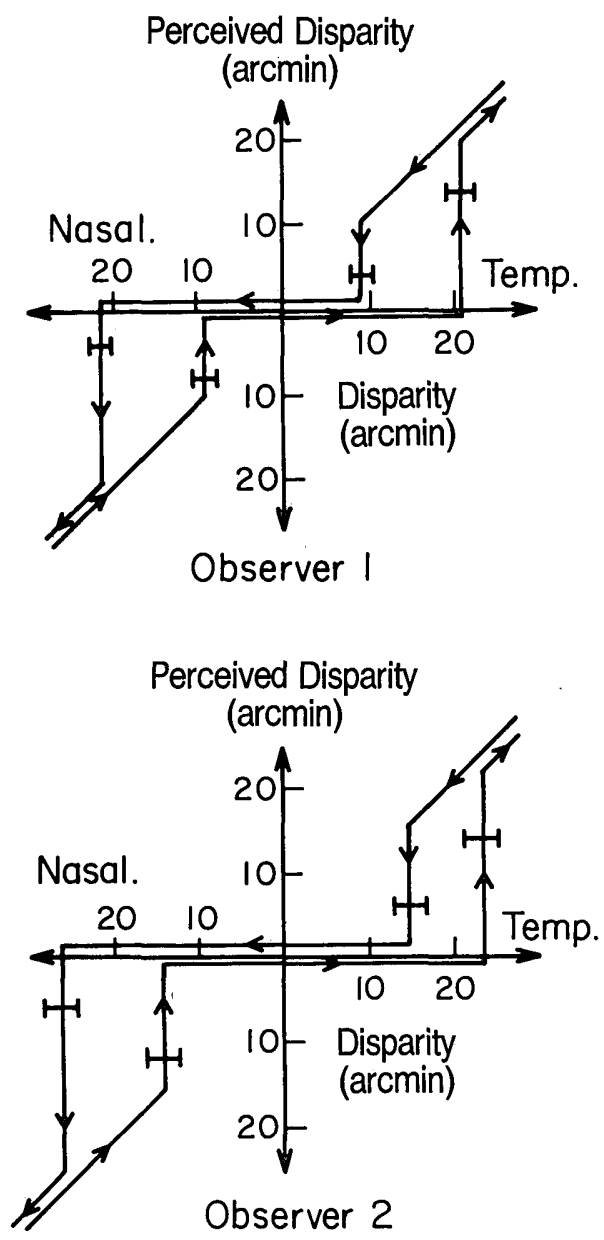

Fig. 3. Same as Fig. 2, but reticules were not used. The right-hand side of each of these diagrams is comparable with Fig. 1, except for standard deviation versus standard error.

of nasalward fusion and the mean value of temporalward fusion never differed by more than 2 arcmin.

The principal objective of this investigation was to compare the nasalward and temporalward ranges of fusion and of the extension of fusion. The ranges of hysteresis and the ranges of fusion that we measure are smaller than those reported by Fender and Julesz ${ }^{4}$ for bar targets; however, the ratios of the ranges of hysteresis to the ranges of fusion that we measure are larger. Therefore we conclude that whether the nasalward or temporalward visual fields are tested has no effect on the magnitude of the hysteresis. The use of reticules may reduce the ranges of fusion, and it surely reduces the hysteresis. The symmetry of motion does not affect the ranges of fusion.

\section{DISCUSSION}

The results of the experiments reported above affirm that Panum's fusional area can be extended into regions of crossed disparity as well as into the regions of uncrossed disparity and that the ranges of extension are symmetric. This symmetry was assumed by Fender and Julesz, ${ }^{4}$ but they did not test it. The oculomotor system can converge the visual axes to compensate for almost any amount of crossed disparity, whereas the divergent motion of the visual axes is severely limited. Thus the remapping system that permits the extension of Panum's fusional area might have developed with asymmetric properties, but our data indicate that this is not true: the remapping system is symmetric.

Both our observers demonstrated fusional ranges significantly smaller than those measured by Fender and Julesz; however, we recently found one observer, whose results are not used in this paper, who is able to fuse the bar targets by voluntary efforts over ranges similar to that reported by Fender and Julesz. ${ }^{4}$ Thus it is possible that this difference can be ascribed to normal observer variability.

The use of the unstabilized circular mask in the present experiments may also affect the range of the phenomenon; Fender and Julesz ${ }^{4}$ did not use such a mask. Their targets were made as photographic 2-in. (approx. 5 -cm) slides; the edges of the slides were visible about $3^{\circ}$ peripherally and may have served as auxiliary fusional cues. This interpretation would agree with the results of Kertesz. ${ }^{8}$

Both of our observers perceived the bars in depth when the reticules were present, and both observers lost the percept of depth when the reticules were absent. The latter result agrees with the observations of Fender and Julesz, ${ }^{4}$ Erkelens and Collewijn, ${ }^{6}$ and Hyson et al. ${ }^{5}$

In general, in order to produce a disparity signal, it is necessary to form at least two images on each retina. Two matching images, one from each retina, are normally brought together by any combination of vergence eye movements and neural processes, and then the visual angle between the other pair of images gives the disparity signal. The data manipulation in this paper has so far used the average of the mean locations of temporalward and nasalward fusion as a statistical approximation to the first pair of retinal images. This procedure allowed us to calculate the disparity directly as the angle between the images of the two bars. But we could also use the images of the reticules as the first pair of images, and in fact this is what the observers were trained to do. However, the retinal images of the reticules were not stabilized. They moved irregularly on the retinas, and Fender and Julesz ${ }^{4}$ have shown that, for nonstabilized images, this motion may account for changes in disparity having a standard deviation of about 8 arcmin.

We might predict therefore that the observer would perceive a bar that advances or retreats in front of and behind the fused percept of the reticules with a motion that has dynamics similar to the dynamics of eye motions during fixation. The observers did not report this percept. They clearly saw a stable bar in front of or behind a percept of a stable reticule. It appears therefore that whatever mechanism holds our percept of the visual world stable in space despite the retinal image motions caused by eye movements, it does not unseat the binocular depth percept caused by stabilized images formed on the same retinas. This suggests that the cortical correction for eye movements, efference copy perhaps, is a signal of intermediate magnitude and that it may not be strong enough to override the neural signals of the depth assigned to the stabilized images.

It is possible that an astute observer could use the time lapse from the percept of temporalward break as an additional cue to guess the time of nasalward fusion. We minimized this possibility by moving the bars to a total disparity of \pm 70 arcmin, that is, well beyond the location of break or fusion. The average minimum elapsed time between one percept of fusional break and the following percept of fusion 
was of the order of $50 \mathrm{sec}$. The exact point of reversal was imperceptible to the observers. The largest hysteresis that we measured was below 12 arcmin; hence a malicious observer would need to estimate the periods of $50 \mathrm{sec}$ with a reliability of $\pm 5 \mathrm{sec}$ if he or she wished to use elapsed time as a fusional cue in order to fool the experimenters. We tested one of our observers in this regard, and he was quite unable to maintain this precision while attending to the other demands of the experiment.

\section{Fusion versus Rivalry}

One of the major difficulties in this type of research is the distortion of the results that can be attributed to the observer's confusing the singleness of the percept of the binocular stimulus caused by rivalry with the singleness of the percept caused by fusion. We paid careful attention to this difficulty and approached it in the following manner. We adopted a strict definition of fusion and instructed our observers to report fusion only if all the following criteria were satisfied:

(1) Only one bar was perceived.

(2) The binocular bar was seen in an intermediate position between the monocular positions of the bars.

(3) The motion of the binocular bar was the combination of the monocular motions of the bars.

(4) The binocular bar was perceived in depth relative to the reticules when present.

During the training sessions, our observers reported two other percepts. First, a fused, retinally stabilized, dark bar on a light background appeared to be darker than a single bar and heavier, as if drawn in India ink. The single, unfused, stabilized bar seemed thinner, lighter, and not so solid as a fused bar. Perhaps this is the percept that Duwaer and van den Brink ${ }^{15}$ describe as restless. Thus we added a fifth criterion:

(5) The binocular bar was perceived as solid, dark, and heavy.

The second report was of a strong percept of diplopic depth just before fusion and immediately after loss of fusion. We did not use this as a criterion for fusion. However, the observers used it to help to distinguish between rivalry and fusion. One or more of these cues was operative in each of our experimental conditions, and our observers, both of whom were experienced visual observers and knowledgeable concerning the objectives of the experiment, were usually able to spot a rivalrous condition and to warn the experimenter. In such a case the datum obtained was excluded.

\section{Fusion versus Fading of Stabilized Images}

Stabilized retinal images tend to fade out; they gradually lose contrast until they are no longer perceived. If the percept of one bar were to fade, then only one bar would remain visible in our paradigm, and problems similar to those caused by rivalry would occur. We dealt with this problem in the same way that we dealt with rivalry.

Despite all our precautions and the cooperation of our observers, rivalry and fading are insidious enemies in this form of experimentation. We cannot rule out the possibility that our data are contaminated by these problems to some extent. However, any error caused by rivalry or by fading would bias our values in the direction of larger values for fusion and for hysteresis, whereas our measured ranges of fusion are smaller than those of all other researchers who use retinally stabilized bar images; so perhaps we have controlled our experiments better than most against contamination by rivalry and fading.

\section{CONCLUSION}

The nasalward limits of Panum's fusional area and the hysteresis demonstrated by the nasalward limits do not differ significantly from the temporalward limits and the hysteresis demonstrated by the temporalward limits. Piantanida ${ }^{7}$ implies that these regions are unequal. However, we believe that this conclusion is bound up with his definition of a preferred setting. It is highly improbable that the position of most comfortable fusion of stabilized random-dot stereograms will be the position of zero disparity. This position is much more likely to be the convergence condition required for a comfortable reading distance and hence "well to the nasal side of the fusional range," as Piantanida reports.

The limits of Panum's fusional area and the hysteresis demonstrated by these limits are the same if one stimulus moves across each retina or if one stimulus is held still on one retina and another stimulus is moved across the other retina.

The use of nonstabilized reticules for fixation modified some of the results, in general reducing the magnitude of the hysteresis.

\section{ACKNOWLEDGMENTS}

We thank B. Julesz for participating in many discussions during the progress of this work. This research was supported in part by grants NS03627, EY02890, EY00085, and RR07003 from the National Institutes of Health.

* Present address, Automated Systems Section, Jet Propulsion Laboratory, California Institute of Technology, Pasadena, California 91109.

\section{REFERENCES}

1. C. Wheatstone, "Contributions to the physiology of vision: Part 1. On some remarkable and hitherto unobserved phenomena of binocular vision," Philos. Trans. 128, 371-394 (1838).

2. H. W. Dove, "Über Stereoskopie," Ann. Phys. Ser. 2 110, 494$498(1860)$.

3. P. L. Panum, Physiologische Untersuchungen über das Sehen mit zwei Augen (Schwerssche Buchhandlung, Kiel, 1858).

4. D. Fender and B. Julesz, "Extension of Panum's fusional area in binocularly stabilized vision," J. Opt. Soc. Am. 57, 819-830 (1967).

5. M. T. Hyson, B. Julesz, and D. H. Fender, "Eye movements and neural remapping during fusion of misaligned random-dot stereograms," J. Opt. Soc. Am. 73, 1665-1673 (1983).

6. C. J. Erkelens and H. Collewijn, "Eye movements in relation to loss and regaining of fusion of disjunctively moving random-dot stereograms," Human Neurobiol. 4, 181-188 (1985).

7. T. P. Piantanida, "Stereo hysteresis revisited," Vision Res. 26, 431-437 (1986).

8. A. E. Kertesz, "Effect of stimulus size on fusion and vergence," J. Opt. Soc. Am. 71, 289-293 (1981).

9. J. J. Kulikowski, "Limit of single vision in stereopsis depends on contour sharpness," Nature 276, 126-127 (1978). 
10. C. Schor, I. Wood, and J. Ogawa, "Binocular sensory fusion is limited by spatial resolution," Vision Res. 24, 661-665 (1984).

11. C. M. Schor and C. W. Tyler, "Spatio-temporal properties of Panum's fusional area," Vision Res. 21, 683-692 (1981).

12. D. S. Gilbert and D. H. Fender, "Contrast thresholds measured with stabilized and non-stabilized sine-wave gratings," Opt. Acta 16, 191-204 (1969).
13. G. J. St-Cyr and D. H. Fender, "Nonlinearities of the human oculomotor system: time delays," Vision Res. 9, 1491-1503 (1969).

14. K. N. Ogle, Researches in Binocular Vision (Hafner, New York, 1950).

15. A. L. Duwaer and G. van den Brink, "What is the diplopia threshold?” Percept. Psychophys. 29, 295-309 (1981). 